\title{
Use of the Unroofing Technique for AFM Direct Imaging of the Intra-Cellular Structure at High Resolution
}

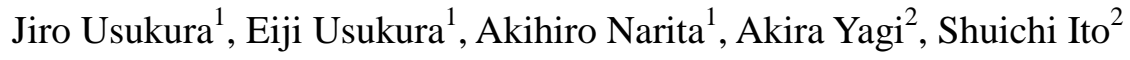 \\ 1. Graduate School of Science, Nagoya University, Nagoya, 464-8630 Japan \\ 2. Olympus Corporation. Hachioji, Tokyo 192-8512 Japan
}

Atomic force microscope (AFM) is capable of detecting topological surface structure of living cells in aqueous condition, but not able to describe inside of the cells directly. Recently, however, unroofing technique provided the way to investigate the intra cellular structure by AFM, since inside of cell was exposed by removing the cell membrane (unroofing) and subsequent washing the cytoplasm out (1). In addition to such improvement of preparation methods, recent function of AFM is advanced markedly, in particular, on resolution and scanning speed. Then we used AFM in order to investigate intracellular cytoskeleton in the buffer solution at high resolution comparable to electron microscopy.

NRK or Vero cells were cultured on the slide glass with hydrophobic framework for a few days. Cultured cells were washed once with HEPE based Ringer's solution and soaked briefly in poly-L-lysine solution followed by washing three times with Ca free Ringer's solution. Subsequently, cells placed in KHMgE buffer (30 mM HEPES, pH 7.4, $70 \mathrm{mM} \mathrm{KCl,} 3 \mathrm{mM} \mathrm{MgCl}$, $1 \mathrm{mM}$ EGTA, 1 mM DTT, $0.1 \mathrm{mM}$ AEBSF (4-(2-aminoethyl) benzenesulfonyl fluoride hydrochloride)), and the dorsal cell membrane was removed (unroofing) by sonication induced bubble jet (2) at low power $(27 \mathrm{kHz}, 0.5 \mathrm{~W})$. Cells unroofed were washed briefly in fresh KHMgE buffer, and then fixed with $2 \%$ glutaraldehyde in the same buffer. After being washed twice with the same buffer, cells unroofed on above slide glass were brought into AFM (BIXAM, Olympus Corporation) for observation.

We succeeded to view membrane cytoskeleton, clathrin coats and caveoli directly in buffer solution at high resolution similar to electron microscope. Spatial architecture of stress fibers formed of single actin filament extending from the cytoplasmic surface of the cell membrane was arrested in detail (Fig 1). In practice, several substances raping and thereby bundling actin filaments was recognized clearly on the stress fibers. AFM was also able to describe characteristic short periodicity of $5 \mathrm{~nm}$ as a striation on the single actin filaments branched from stress fibers. Furthermore, clathrin coated pits and caveoli in addition to peripheral membrane proteins were observed clearly. In common sense, it has been considered to be difficult so far that AFM displayed the surface of caveoli in detail, though characteristic vortex ridges (supposed to be caveolin 1) appeared on the surface of caveola were detected clearly rather than in freeze-etching replica and scanning electron microscopy (Fig. 2). This is due in part to loss of metal coating. In case of clathrin coats, frequently, tri-skelion of clathrin and adaptin were partly discernible (Fig. 3). Fig.4 shows higher magnification image of unfixed single actin filament with periodical striations of $5 \mathrm{~nm}$ that are derived from assembled G actins. Thus, isolated single actin fibers were observed well at high resolution under non-fixed condition. However, in the cells, it is difficult to observe the single actin filament at atomic resolution since unknown reason.

Spatial architecture of cytoskeleton, in particular actin filaments crowing cytoplasmic membrane surface, clathrin coats and caveoli were successfully observed directly in buffer solution under AFM at high resolution comparable to electron microscope. 


\section{References:}

[1] Usukura J et al. J. Electron Microsc. 61 321-326 2012

[2] Heuser, J E, Anderson, R G J. Cell Biol. 108 (1989), 389-400

[3] The author (J.U) acknowledges funding from the Japan Science and Technology Agency (JST),

"Development of Systems and Technologies for Advanced Measurement and Analysis" Program.
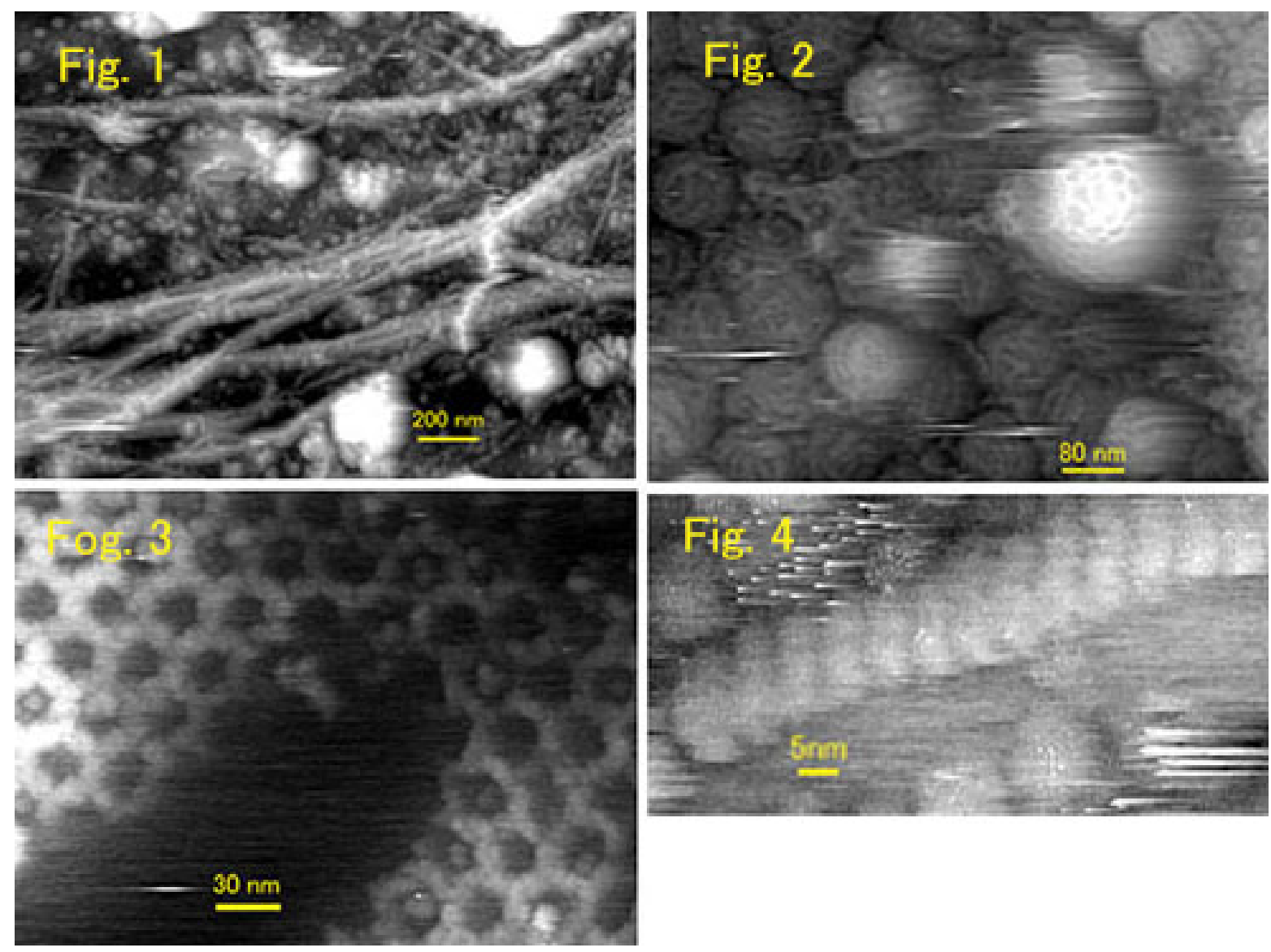

Fig.1: Membrane cytoskeleton showing stress fiber and branching actin filaments on the cell membrane. Fig. 2: Cluster of Caveola showing characteristic ridge structures on their surface.

Fig.3: High power image of clathrin coat. Fig. 4: Molecular resolution image of an isolated actin filament. 